\title{
INFLUÊNCIA DE DIFERENTES SOLVENTES NO CONTEÚDO DE COMPOSTOS FENÓLICOS EM EXTRATOS DE CASCAS DE UVA (VITIS VINIFERA) DA VARIEDADE SYRAH
}

\author{
Euzélia Lima de Souza1; Talita Sousa Nascimento2; Gabriele de Abreu Barreto ${ }^{3}$; Ingrid \\ Lessa Leal ${ }^{3}$; Bruna Aparecida Souza Machado ${ }^{3,4}$. \\ ${ }^{1}$ SENAI CIMATEC - Programa de Pós-graduação em Gestão e Tecnologia Industrial \\ - Salvador/Ba. \\ 2Universidade Federal da Bahia, Escola de Nutrição - Salvador/Ba. \\ ${ }^{3}$ SENAI CIMATEC - Laboratório Biotecnologia e Alimentos - Salvador/ Ba \\ ${ }^{4}$ SENAI CIMATEC - Instituto de Tecnologias da Saúde (ITS) - Salvador/Ba.
}

Resumo: A casca de uva proveniente da indústria de vinho ainda contém expressivas quantidades de compostos fenólicos. Características especificas dos solventes aplicados influenciam diretamente na extração de compostos fenólicos. O objetivo do presente trabalho foi avaliar a influência de diferentes solventes na obtenção de compostos fenólicos em extratos da casca de uva Syrah por cromatografia líquida de alta eficiência. Foram elaborados extratos de cascas de uvas com 3 diferentes solventes (água, álcool de cereais a 50\% e $80 \%$ ), e avaliados utilizando curva padrão com 15 compostos fenólicos. Não foi possível quantificar os compostos fenólicos, porém os cromatogramas sugerem a presença de alguns deles. O solvente que proporcionou melhor interação com a casca de uva foi o álcool de cereais a $50 \%$.

Palavras-Chave: antioxidante, resíduo, casca de uva, cromatografia.

\section{INFLUENCE OF DIFFERENT SOLVENTS ON THE PHENOLIC COMPOUNDS CONTENT GRAPE EXTRACTS (VITIS VINIFERA) FROM SYRAH VARIETY}

\begin{abstract}
Grape rind from the wine industry still contains significant amounts of phenolic compounds. Specific characteristics of the applied solvents directly influence the extraction of phenolic compounds. The objective of this work was to evaluate the influence of different solvents on phenolic compounds in Syrah grape husk extracts by high performance liquid chromatography. Grape peel extracts were prepared with 3 different solvents (water, $50 \%$ and $80 \%$ cereal alcohol), and evaluated using standard curve with 15 phenolic compounds. It was not possible to quantify the phenolic compounds, but the chromatograms suggest the presence of some of them. The solvent that provided the best interaction with grape peel was 50\% cereal alcohol.
\end{abstract}

Keywords: antioxidants, residue, grape peel, chromatography. 


\section{INTRODUÇÃO}

Diversas pesquisas ao longo do tempo têm demostrado interesse em estudar os efeitos benéficos à saúde atribuídos aos compostos fenólicos presentes na maioria dos alimentos. Os estudos destacam os múltiplos efeitos biológicos e clínicos relacionados a estes compostos da dieta como papeis antioxidantes, cardioprotetor, anticâncer, anti-bacteriano, anti-virais, vasodilatador, antidiabética e ações antiinflamatórias [1].

Os compostos fenólicos, constituem um dos maiores grupos de metabolitos secundários das plantas com mais de 8000 já conhecidas. Eles são importantes constituintes dos alimentos. As uvas contem expressivas quantidades desses compostos quando comparada a outras frutas e vegetais [2]. O teor total de fenólicos das cascas de uva variam conforme a cultivar, fatores como topografia, geologia, drenagem, clima, microclima, castas, intervenção humana (terroir) [3].

Pesquisas apresentam grande eficácia em reutilização do bagaço da uva por conter expressivas quantidades de fenólicos e antioxidantes. Na casca são encontrados componentes como os flavonóis (quempferol, quercetina e miricetina) [4], as antocianinas (cianidina, delfinidina, peonidina, petunidina, malvidina), os estilbenos (resveratrol), os ácidos fenólicos (ácido cafeiltartárico e o ácido p-cumariltartárico), além de uma larga variedade de taninos [5-7].

Para a extração de compostos fenólicos de diversas matrizes, diferentes sistemas de solventes são utilizados [8]. A extração por solventes é o método mais comum para separar antioxidantes naturais. Estes quando em série nos alimentos, com solventes diversificados, pode ser mais eficiente na extração de compostos bioativos, uma vez que leva em conta a diversidade de cada amostra [9]. O sistema solvente utilizado na extração de bagaço de uva influencia diretamente os conteúdos de fenólicos totais, antocianinas e atividade antioxidante dos extratos [6].

O tipo e a polaridade do solvente são características que afetam a transferência de elétrons e de átomos de hidrogênio, aspecto-chave na extração de polifenois, e consequentemente na capacidade antioxidante [10]. Além do rendimento, a escolha do sistema solvente a ser utilizado também influencia na composição do extrato [11].

Considerando a importância dos compostos fenólicos para a saúde humana, o objetivo do presente trabalho foi avaliar a influência de diferentes solventes na obtenção de compostos fenólicos em extratos da casca de uva Syrah por cromatografia líquida de alta eficiência.

\section{METODOLOGIA}

\subsection{Material e Reagentes}

Foram utilizados metanol, DMSO (Sigma-Aldrich Chemical Co. - St. Louis, MO, EUA) e ácido acético (Dinâmica) de grau HPLC, e álcool de cerais (92,8\% - Anidrol). Utilizou-se filtro para seringa de nylon de 0,20 $\mu$ m (Agilent Captiva, Califórnia, EUA). Os padrões ácido cafeico (CAS number 331-39-5), ácido gálico (CAS number 14991-7), ácido trans-cinâmico (CAS number 205-398-1), ácido p-cumárico cristalino (CAS number 501-98-4), biochanina A (CAS number 91-80-5), catequina (CAS 


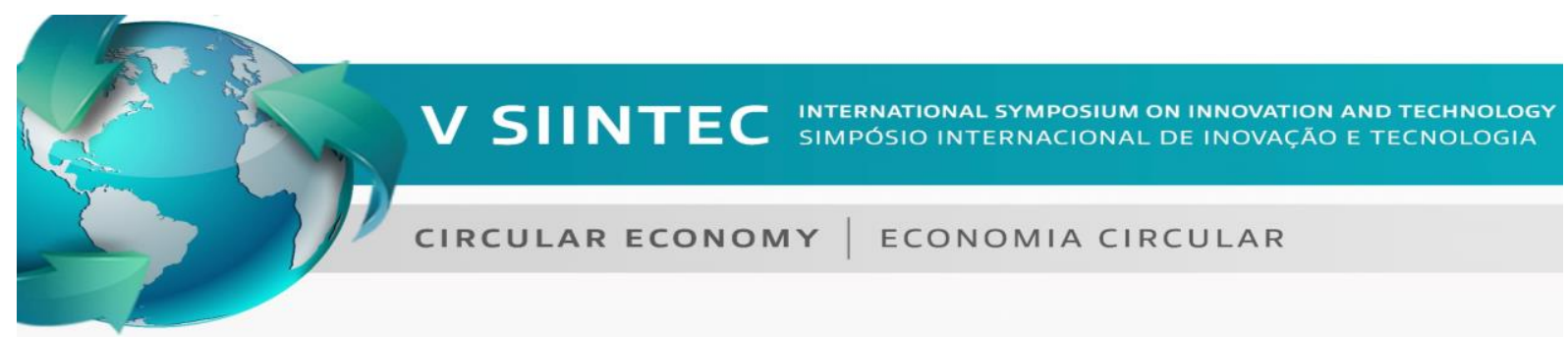

number 7295-85-4), epicatequina (CAS number 490-46-0), formononetina (CAS number 485-72-3), isoliquiritigenina (CAS number 961-29-5), miricetina (CAS number 529-44-2), narigenina (CAS number 67604-48-2), quercetina (CAS number 117-39-5), resveratrol (CAS number 501-36-0) e rutina hidratada (CAS number 207671-50-9) foram adquiridos da Sigma-Aldrich Chemical Co. (St. Louis, MO, EUA) e ácido transferúlico (CAS number 537-98-4) da Fluka.

\subsection{Obtenção dos Extratos}

As amostras de bagaço de uva Syrah (Vitis vinifera L.) utilizadas foram obtidas de indústrias vitivinícolas da região do Vale do São Francisco no mês de agosto/2018. As cascas foram separadas da semente e engaço e mantidas sob congelamento $(-20$ $\left.{ }^{\circ} \mathrm{C}\right)$ até o momento de uso. Foram obtidos 3 extratos de casca de uva Syrah com variação do solvente: extrato aquoso (EA), extrato de álcool de cereais a 50\% (EAC50) e extrato de álcool de cereais a 80\% (EAC80). As cascas foram trituradas com o solvente na proporção 1:5 m/v. Em seguida, a mistura foi sonicada (Elma Sonic, $\mathrm{S} 40 \mathrm{H}$, Alemanha), por $30 \mathrm{~min} / 60^{\circ} \mathrm{C}$. Posteriormente, foi homogeneizada em incubadora tipo shaker por $120 \mathrm{~min}$ (180 rpm), filtrada e concentrada em concentrador de amostra (Genevac, MiVac Concentrator, Reino Unido), a $50^{\circ} \mathrm{C}$. O extrato foi armazenado em temperatura de congelamento $\left(-27^{\circ} \mathrm{C}\right)$.

\subsection{Análise Cromatográfica dos Extratos}

Para a identificação e quantificação dos compostos (Ácido Cafeico, Ácido Gálico, Ácido p-cumárico, Ácido Trans-cinâmico, Ácido Trans-ferúlico, Biochanina A, Catequina, Epicatequina, Formonometina, Isoliquiritigenina, Miricetina, Naringenina, Quercetina, Resveratrol e Rutina Hidratada), inicialmente foram preparadas soluções dos padrões à concentração de $10 \mathrm{mg} \cdot \mathrm{L}^{-1}$, os quais foram dissolvidos em metanol de elevada pureza. Soluções metanólicas de extratos de uva também foram preparadas (5500 ppm). As amostras foram filtradas em filtro de nylon de $25 \mathrm{~mm}, 0,20 \mu \mathrm{m}$ (Agilent Captiva) para injeção em Cromatógrafo Líquido de Alta Eficiência (HPLC) (Shimadzu, LC-20AT, Japão) equipado com injetor automático e detector de arranjo de diodo (DAD) (Shimadzu, SPD-M20, Japão). A separação cromatográfica foi adaptada tendo por base o método proposto por Salgueiro \& Castro [12] e Cabral et al. [13]. A coluna NUCLEODUR® 100-5 C18 ec (150x4 mm ID; tamanho de partícula de $5 \mu \mathrm{m})$ foi utilizada em conjunto com uma pré-coluna ZORBAX Eclipse Plus C18 4,6x12,5 mm (Agilent, USA).

As condições de análise foram realizadas com um gradiente de eluição com uma fase móvel de ácido acético $5 \%$ e metanol em diferentes proporções e com tempo de análise total de 42 minutos (de 0 a $35 \min (0-92 \%$ B); 35 a 40 min $(92-0 \%$ B); 40 a $42 \min (0 \% \mathrm{~B}))$. $\mathrm{O}$ volume de injeção foi de $20 \mu \mathrm{L}$ e o fluxo de $1 \mathrm{~mL} \cdot \mathrm{min}^{-1}$. $\mathrm{O}$ equipamento foi operado à temperatura de $40 \pm 2{ }^{\circ} \mathrm{C}$. A leitura do detector de arranjo de diodos foi ajustada na faixa de 190 a $800 \mathrm{~nm}$ e a aquisição cromatográfica foi definida entre 260 e $370 \mathrm{~nm}$. A identificação dos compostos foi realizada através da comparação do tempo de retenção e do espectro ultravioleta entre as amostras e os padrões (Tabela 1), a faixa de trabalho dos compostos foi de 0,5 a $30 \mathrm{mg} \cdot \mathrm{L}^{-1}$. 
Tabela 1. Limite de detecção e quantificação dos compostos ativos por HPLC DAD

\begin{tabular}{|c|c|c|c|c|}
\hline Composto Fenólico & $\underset{(\min )}{t R}$ & $\stackrel{\wedge}{(\mathrm{nm})}$ & $\begin{array}{l}\text { Limite de } \\
\text { Detecção } \\
\left(\mathrm{mg}^{-1}\right)\end{array}$ & $\begin{array}{c}\text { Limite de } \\
\text { Quantificação } \\
\left(\mathrm{mg}^{-1} \mathrm{~g}^{-1}\right)\end{array}$ \\
\hline Ácido Cafeico & 8,20 & 320 & 0,29 & 0,96 \\
\hline Ácido Gálico & 2,32 & 280 & 0,96 & 3,21 \\
\hline Ácido p-cumárico & 10,35 & 300 & 1,04 & 3,48 \\
\hline Ácido Trans-cinâmico & 16,02 & 280 & 0,28 & 0,93 \\
\hline Ácido Trans-Ferúlico & 11,33 & 320 & 1,99 & 6,64 \\
\hline Biochanina $\mathrm{A}$ & 22,00 & 280 & 0,41 & 1,38 \\
\hline Catequina & 6,59 & 280 & 1,07 & 3,58 \\
\hline Epicatequina & 8,45 & 280 & 0,81 & 2,69 \\
\hline Formononetina & 19,28 & 300 & 0,28 & 0,94 \\
\hline Isoliquiritigenina & 18,70 & 370 & 0,34 & 1,14 \\
\hline Miricetina & 13,06 & 370 & 0,72 & 2,39 \\
\hline Naringenina & 16,54 & 280 & 0,24 & 0,79 \\
\hline Quercetina & 15,30 & 370 & 0,77 & 2,57 \\
\hline Resveratrol & 13,79 & 300 & 0,28 & 0,93 \\
\hline Rutina Hidratada & 11,23 & 260 & 0,29 & 0,97 \\
\hline
\end{tabular}

tR=tempo de retenção; $\Lambda=$ comprimento de onda; $L D=$ limite de detecção; $L Q=$ limite de quantificação.

Com o objetivo de garantir a confiabilidade dos resultados obtidos, foi realizada uma validação de acordo com as metodologias da Agência Nacional de Vigilância Sanitária (ANVISA) [14] e do Instituto Nacional de Metrologia, Qualidade e Tecnologia (INMETRO) [15]. Esta análise foi realizada de acordo com os parâmetros de seletividade, linearidade, precisão, limites de detecção e limites de quantificação.

\section{RESULTADOS E DISCUSSÃO}

Os compostos fenólicos investigados nos diferentes extratos de casca uvas são apresentados na Tabela 2.

Tabela 2. Avaliação qualitativa dos compostos ativos presentes em extratos de uva Shiraz obtidos com diferentes solventes

\begin{tabular}{cccc}
\hline Composto fenólico & EA & EAC50 & EAC80 \\
\hline Ácido Cafeico & $<$ LD & $<$ LQ & $<L Q$ \\
Ácido Gálico & ND & $<$ LD & $<L D$ \\
Ácido p-cumárico & $<L D$ & $<L D$ & $<L D$ \\
Ácido Trans-cinâmico & $<L D$ & $<L Q$ & $<L D$ \\
Ácido Trans-Ferúlico & ND & $<L D$ & $<L D$ \\
Biochanina A & ND & ND & $<L D$ \\
Catequina & $<L D$ & ND & $<L D$ \\
Epicatequina & $<L D$ & $<L D$ & $<L D$ \\
Formononetina & ND & ND & ND \\
Isoliquiritigenina & ND & ND & ND \\
Miricetina & $<L D$ & $<L Q$ & $<L Q$ \\
Naringenina & $<L D$ & $<L D$ & $<L D$ \\
Quercetina & ND & ND & ND
\end{tabular}


Resveratrol Rutina Hidratada
$\begin{array}{lll}N D & <L D & <L D \\ <L D & <L Q & <L Q\end{array}$

$N D=N a ̃ o$ detectado; $\angle L D=$ abaixo do limite de detecção; $\angle L Q=$ abaixo do limite de quantificação

Ressalta-se que apesar de nenhum compostos ser quantificado, os extratos apresentaram em seus cromatogramas picos que sugerem a presença de alguns compostos em sua composição fitoquímica, sendo estes sinalizados com <LD (abaixo do limite de detecção) na Tabela 2.

De acordo com os resultados apresentados, sugere-se que o solvente que proporcionou melhor interação com a casca de uva foi o álcool de cereais a $50 \%$. Abaixo tem-se o cromatograma (Figura 1) para a rutina no comprimento de onda de 260nm.

Figura 1. Cromatograma dos extratos de uva obtidos com diferentes solventes. EA (A), EAC50 (B) e EAC80 (C)
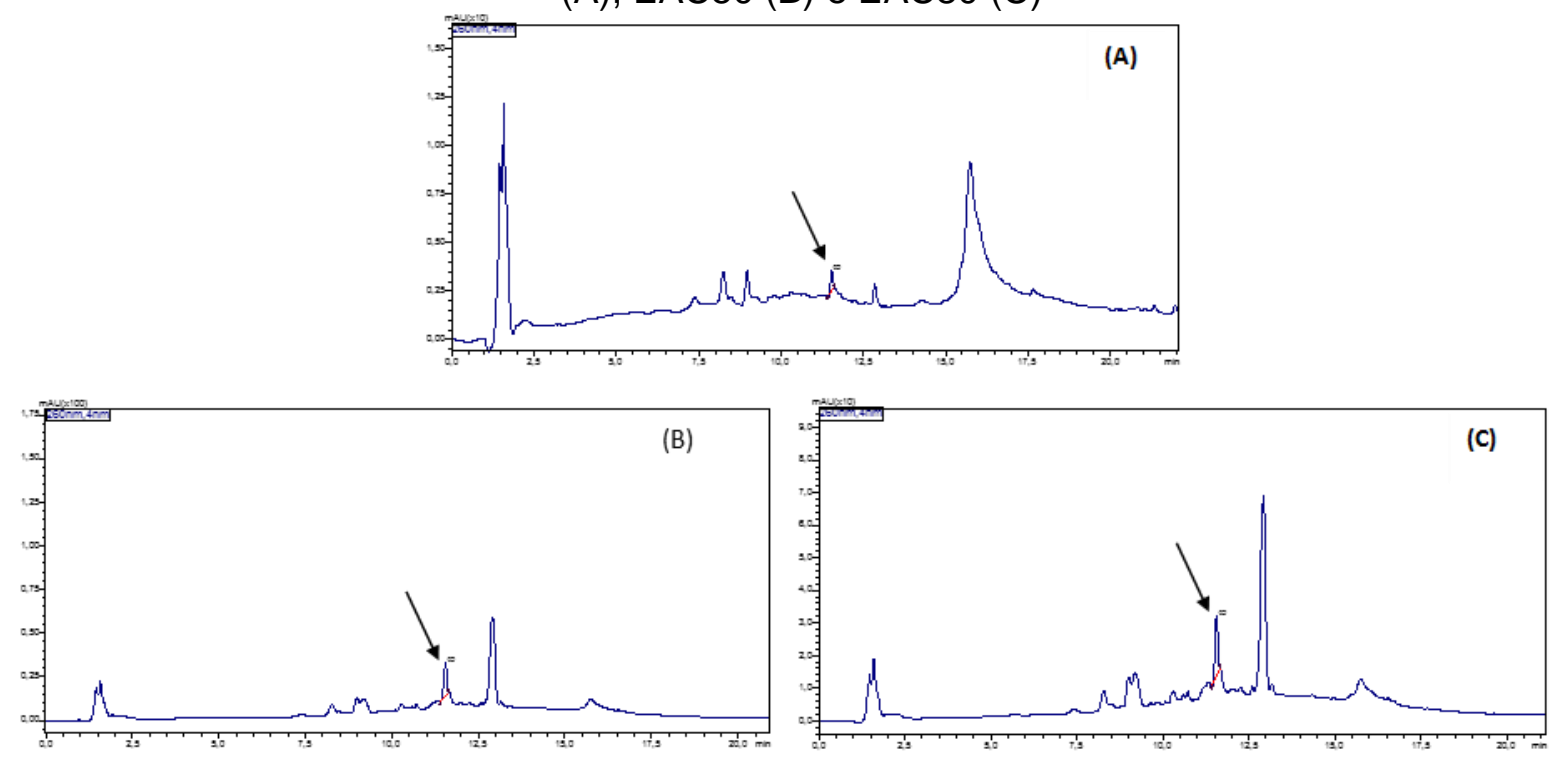

A miricetina é um isoflavanol com atividade antioxidante, é comumente distribuída em frutas, vegetais, nozes, frutas vermelhas, chá e vinho tinto [16]. Acredita-se que mirecetina apresenta múltiplos efeitos terapêuticos, potenciais propriedades para saúde como antioxidante [17,18], anti-carcinogênicas, antiinflamatórias, anti-ateroscleróticas, anti-trombóticas, anti-diabéticas e antivirais [19], exercem efeitos anti- e prooxidantes, bem como exibem efeitos mutagênicos e potencial anti-mutagênico, [18,20-22].

A rutina, flavona natural, apresenta diversas propriedades, incluindo antialérgica, anti-inflamatória, antitumoral e principalmente antioxidante, antidiarreico, antihipertensivo, antimutagênica e proteção contra o estresse nitrosativo e lesão hepatocelular $[18,23,24]$.

Wang et al. [25] ao analisar extrato de uva, obtidos com soluções de $\mathrm{HCl}$ e metanol (1:1) e acetona (70\%) e analisados pelo mesmo método cromatográfico encontrou valores de $34,8 \pm 3,3 \mu \mathrm{g}$.mg e $9.0 \pm 0.8 \mu \mathrm{g} . \mathrm{mg}$ para miricetina e rutina. 


\section{CONCLUSÃO}

De acordo com os resultados obtidos, observou-se que os extratos apresentam compostos fenólicos em sua composição, entretanto, a concentração analisada das amostras foi relativamente baixa em comparação com curva analítica utilizada para o método de análise. Desta forma, infere-se que a matriz desde estudo (casca de uva) possui compostos fitoquímicos, porém uma nova análise com uma maior concentração faz-se necessária para quantificar efetivamente estas substâncias.

\section{Agradecimentos}

Os autores agradecem ao CNPQ pela bolsa concedida e ao SENAI CIMATEC, em especial ao Laboratório Integrado e Aplicado em Química (LIPAQ) e ao Laboratório de Biotecnologia e Alimentos.

\section{REFERÊNCIAS}

${ }^{1}$ Pietta P-G. Reviews Flavonoids as Antioxidants. J Nat Prod. 2000;63,: 1035-1042. doi:10.1021/np9904509

2 Vedana MIS, Ziemer C, Miguel OG, Portella AC, Candico LMB. Efeito Do Processamento Na Atividade Antioxidante De Uva. Alim Nutr. 2008;19: 159-165.

${ }^{3}$ Lingua MS, Fabani MP, Wunderlin DA, Baroni M V. In vivo antioxidant activity of grape, pomace and wine from three red varieties grown in Argentina: Its relationship to phenolic profile. J Funct Foods. 2016;20: 332-345.

${ }^{4}$ Beres C, Simas-Tosin FF, Cabezudo I, Freitas SP, lacomini M, Mellinger-Silva C, et al. Antioxidant dietary fibre recovery from Brazilian Pinot noir grape pomace. Food Chem J. 2016;201: 145-152.

${ }^{5}$ Drosou C, Kyriakopoulou K, Bimpilas A, Tsimogiannis D, Krokida M. A comparative study on different extraction techniques to recover red grape pomace polyphenols from vinification byproducts. Ind Crops Prod. 2015;75: 141-149.

${ }^{6}$ Rockenbach II. Compostos fenólicos, ácidos graxos e capacidade antioxidante do Bagaço da vinificação de uvas tintas (Vitis vinifera L. e Vitis labrusca L.). . 112 p.Dissertação (Mestrado em Ciência dos Alimentos) - Centro de Ciências Agrárias,Universidade Federal de Santa Catarina, 2008.

${ }^{7}$ Caldas TW, Mazza KEL, Teles ASC, Mattos GN, Brígida AIS, Conte-Junior CA, et al. Phenolic compounds recovery from grape skin using conventional and nonconventional extraction methods. Ind Crops Prod. 2018;111: 86-91.

${ }^{8}$ Chavan U., Shahidi F, Naczk M. Extraction of condensed tannins from beach pea (Lathyrus maritimus L.) as affected by different solvents. Food Chem. 2001;75: 509512.

${ }^{9}$ Litwinienko G, Ingold KU. Abnormal Solvent Effects on Hydrogen Atom Abstraction. 3. Novel Kinetics in Sequential Proton Loss Electron Transfer Chemistry. J Org Chem. 2005;70: 8982-8990. 
10 Rockenbach II, Lessa Da Silva G, Rodrigues E, Kuskoski EM, Fett R. Influência do solvente no conteúdo total de polifenóis, antocianinas e atividade antioxidante de extratos de bagaço de uva (Vitis vinifera) variedades Tannat e Ancelota. Ciênc Tecnol Aliment. 2008;28: 238-244.

${ }^{11}$ Moure A, Cruz JM, Franco D, Domínguez JM, Sineiro J, Domínguez H, et al. Natural antioxidants from residual sources. Food Chem. 2001;72: 145-171.

12 Salgueiro FB, Castro RN. Comparison between chemical composition and antioxidant activity of different extracts of green propolis. Quim Nova. 2016;39: 11921199.

${ }^{13}$ Cabral ISR, Oldoni TLC, Prado A, Bezerra RMN, Alencar MS. Composição fenólica, atividade antibacteriana e antioxidante da própolis vermelha brasileira. Quim Nova. 2009;32: 1523-1527.

14 BRASIL. Resolução no 899, de 29 de maio de 2003: Guia para validação de métodos analíticos e bioanalíticos. 2003.

15 BRASIL. Diretrizes para Validação de Métodos Analíticos. 2011.

${ }^{16}$ Seo J, Lee D, Arasu M, Wu Q, Suzuki T, Yoon Y, et al. Quantitative differentiation of phenolic compounds in different varieties of buckwheat cultivars from China, Japan and Korea. J Agric Chem Environ. 2013;2: 109-116.

17 Chen W, Feng L, Shen Y, Su H, Li Y, Zhuang J, et al. Myricitrin inhibits acrylamidemediated cytotoxicity in human caco-2 cells by preventing oxidative stress. Biomed Res Int. 2013; 1-7.

18 Domitrovic R, Rashed K, Cvijanovic O, Vladimir-Kneževi S, Škoda M, Višnic A. Myricitrin exhibits antioxidant, anti-inflammatory and antifibrotic activity in carbon tetrachloride-intoxicated mice. Chem Biol Interact. 2015;230: 21-29.

19 Ong KC, Khoo H-E. Biological Effects of Myricetin. Gen Pharmac. 1997;29: 121126.

20 Kim HH, Kim DH, Kim MH, Oh MH, Kim SR, Park KJ, et al. Flavonoid constituents in the leaves of Myrica rubra sieb. et zucc. with anti-inflammatory activity. Arch Pharm Res. 2013;36: 1533-1540.

${ }^{21}$ Meotti FC, Missau C, Ferreira J, Pizzolatti G, Mizuzaki C, Nogueira CW, et al. Antiallodynic property of flavonoid myricitrin in models of persistent inflammatory and neuropathic pain in mice. Biochem Pharmacol. 2006;72: 1707-1713.

22 Shimosaki S, Tsurunaga $\mathrm{Y}$, Itamura H, Nakamura M. Anti-allergic effect of the flavonoid myricitrin from Myrica rubra leaf extracts invitro and invivo. Nat Prod Res. 2011;25: 374-380.

23 Moawad Mahmoud A. Influence of rutin on biochemical alterations in hyperammonemia in rats. Exp Toxicol Pathol. 2012;64: 783-789.

${ }^{24}$ Su K, Yu C, Chen Y, Huang Y, Che C, Hsueh F, et al. Rutin, a flavonoid and principal component of Saussurea involucrata, attenuates physical fatigue in a forced swimming mouse model. Int J Med Sci. 2014;11: 528-537.

25 Wang S, Mateos R, Goya L, Amigo-Benavent M, Sarriá B, Bravo L. A phenolic 
extract from grape by-products and its main hydroxybenzoic acids protect Caco- 2 cells against pro-oxidant induced toxicity. Food Chem Toxicol. 2016;88: 65-74. 